\title{
Isolation Enhancement between Indoor Repeater Antennas with Chip Resistor Embedded FSS
}

\author{
Jae-Yeong Lee, Byeongkwan Kim, Myun-Joo Park, and Byungje Lee \\ Department of Wireless Communications Engineering, Kwangwoon University, 447-1, Wolgye-Dong, Nowon-Gu, \\ Seoul 139-701, Republic of Korea \\ Correspondence should be addressed to Byungje Lee, bj_lee@kw.ac.kr
}

Received 7 November 2011; Accepted 29 November 2011

Academic Editor: Dau-Chyrh Chang

Copyright ( $\odot 2012$ Jae-Yeong Lee et al. This is an open access article distributed under the Creative Commons Attribution License, which permits unrestricted use, distribution, and reproduction in any medium, provided the original work is properly cited.

The isolation enhancement between the donor antenna and the service antenna for indoor repeater systems is presented by using a frequency-selective surface (FSS). A unit cell of the proposed FSS consists of a quarter-wavelength resonator, a chip resistor, an FR4 substrate, and a ground plane. Applying the unit cells of the proposed FSS embedded a chip resistor on the side walls of each reflector for indoor WCDMA repeater antennas and aligning them along with the cross-polarization of each antenna, the isolation is improved by about $13 \mathrm{~dB}$ at the WCDMA band.

\section{Introduction}

In a wireless communication system such as WCDMA $(1.92 \mathrm{GHz}-2.17 \mathrm{GHz})$, when the base station signal transmits from the outdoor environments to the in-buildings or the underground areas, the shadow regions may be generated. Although an indoor WCDMA repeater has been widely used to cover these shadow areas for small coverage, it can cause a self-oscillation between the transmitting signal from the service antenna and the receiving signal to donor antenna when it performs to relay in the identical frequency band [1]. To avoid this mutual interference noise, obtaining a high isolation between closely mounted the donor antenna and the service antenna is one of the most important factors in designing indoor repeater antennas. Figure 1 shows that a conventional indoor repeater system includes two linearly polarized antennas backed by the rectangle-bowl-type reflectors which stand back to back. Two linearly polarized antennas, which are aligned along with the orthogonal (vertical and horizontal) direction to each copolarized component, are positioned at the center of each reflector. Even though two antennas are aligned along with an orthogonal polarization to each other to obtain higher isolation between them, the surface waves on the walls of the reflectors can cause the cross-polarization that leads the unwanted mutual coupling between the antennas. To solve this problem, several kinds of the high impedance surfaces (HIS) have been presented [2-4]. The radar absorbing material structure with a mushroom-like HIS and a chip resistor is proposed $[5,6]$. This structure with a chip resistor is able to provide an absorbing performance and a suppressing surface waves among all transverse fields on the ground surface. However, when this structure is applied to the indoor repeater antenna, it is difficult to maintain the peak gain owing to the degradation of a radiation pattern. The defected ground structure is presented to improve the isolation between a pair of closely packed PIFAs, patches, and monopole antennas on the common ground plane [79]. This structure can obtain a band-stop effect due to the combination of an inductance and a capacitance, so that it applies to antenna designs to suppress the harmonics and the cross-polarizations. However, it is required to redesign the ground plane for conventional indoor repeaters because this structure is etched onto the single ground plane. Without redesigning conventional repeater antennas, we proposed a simple and compact FSS (Frequency Selective Surface), which is embedded into reflectors aligned along with the cross-polarization direction of each antenna to improve the isolation between two antennas for an indoor WCDMA repeater system [10]. 


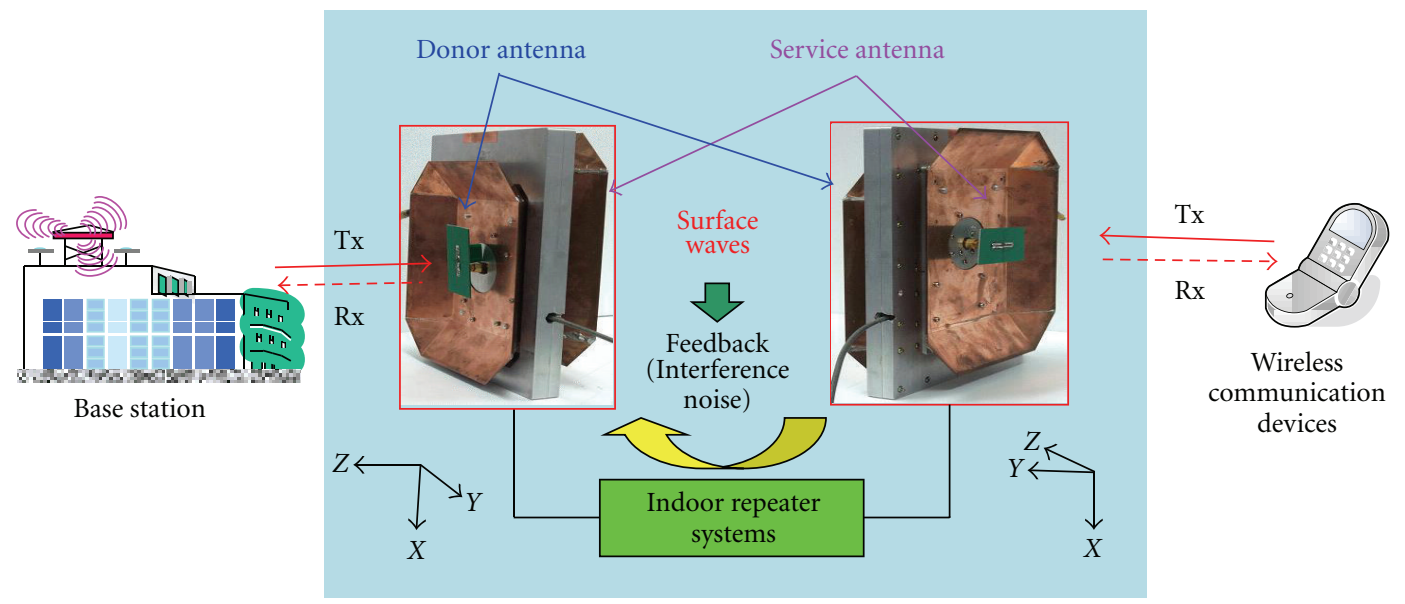

FIGURE 1: Geometry of conventional indoor repeater systems.

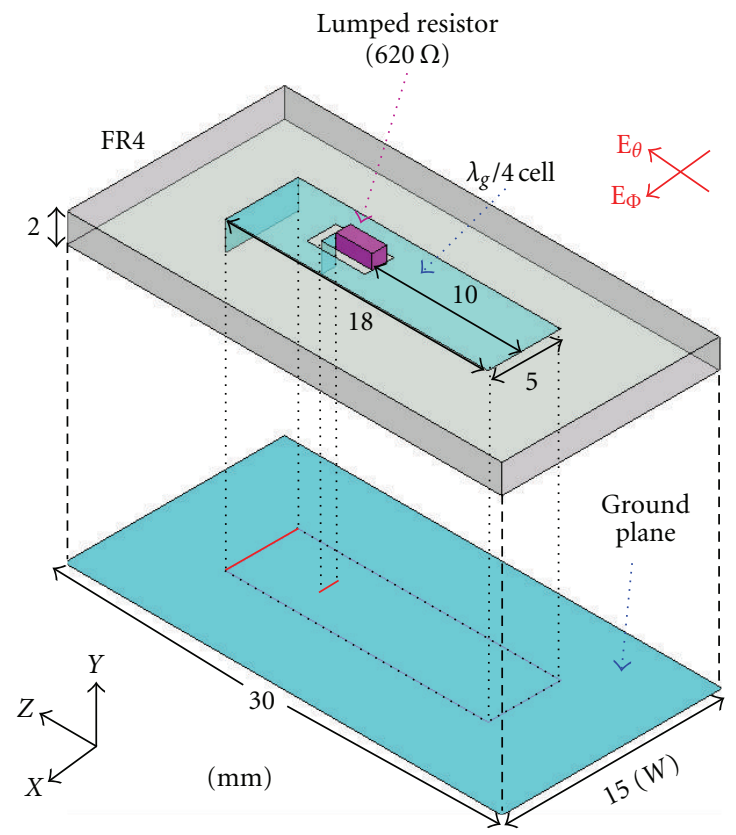

FIgURE 2: Geometry of proposed unit cell of the FSS.

In this letter, a simple unit cell of the FSS where a chip resistor is embedded to act as an absorbing material is proposed to further enhance the isolation between antennas. The unit cell of the proposed FSS is characterized by the HIS and is a quarter-wavelength resonator operated at WCDMA $(1.92 \mathrm{GHz}-2.17 \mathrm{GHz})$ band. To improve the isolation and maintain the peak gain, the unit cell of the proposed FSS is designed to act as the perfect electric conductor for the copolarized component of antennas and act as the HIS for the cross-polarized component of antennas. The proposed unit cells of the FSS are arranged with a periodic structure and are imbedded into the side walls of reflector aligned along with the cross-polarization of each antenna.

\section{Proposed FSS Unit Cell Design}

Typically, two antennas (a donor and a service antenna) for an indoor repeater system stand back-to-back and share the common ground plane (reflectors). This causes poor isolation between them because the surface waves on the reflectors of antennas lead to the unwanted mutual coupling, so that, in this work, the proposed FSS is embedded into the side walls of reflectors. Since the reflector is the rectanglebowl-type and its inclined angle between the wall of reflector and the bottom of reflector is about $45^{\circ}$, the configuration of reflector has to be considered $[11,12]$ when the device suppressing surface waves is designed. The unit cell of the proposed FSS is excited by the obliquely incident wave, so that the surface impedance of the unit cell depends on the inclined angle between the wall of reflector and the bottom of the reflector.

Using the Floquet modal analysis, the surface impedance of the proposed unit cell can be calculated. Basically, Floquet modes are plane waves with propagation direction set by the frequency, phasing, and geometry of the periodic structure. 


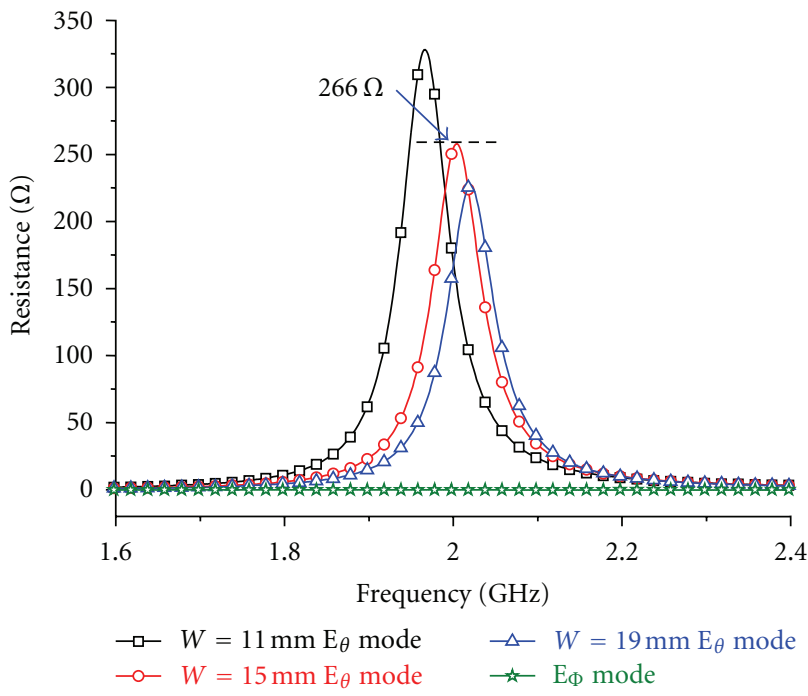

(a)

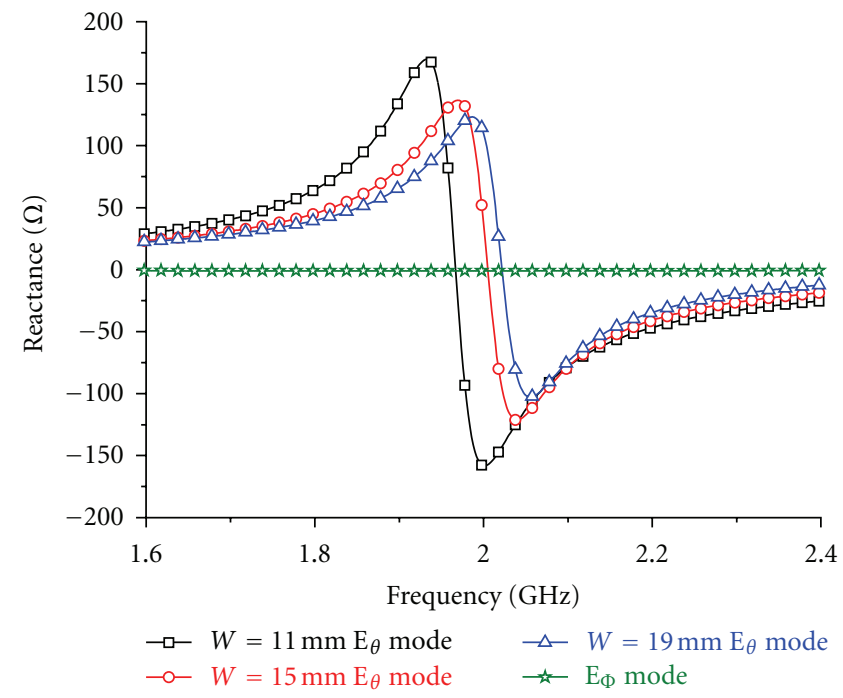

(b)

FIGURE 3: Simulated surface impedance of FSS unit cell in incident mode directions $\left(\mathrm{E}_{\theta}, \mathrm{E}_{\Phi}\right)$ with varying the width (W) of unit cell ground plane: (a) resistance and (b) reactance.

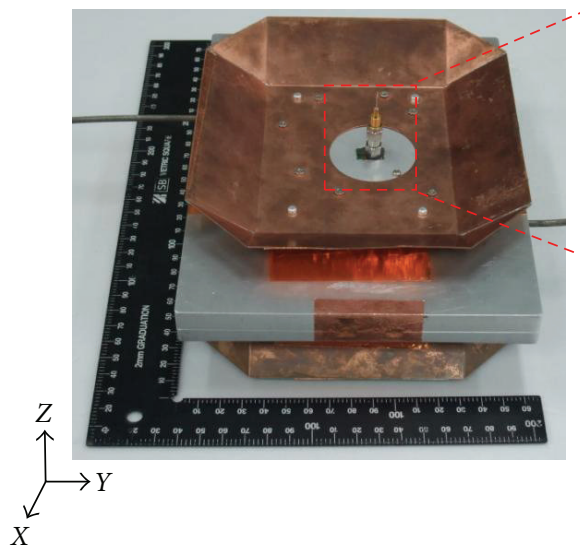

(a)

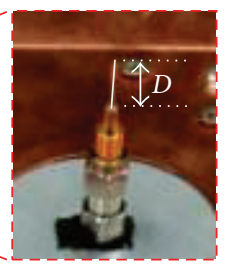

Small monopole $(D=10 \mathrm{~mm} \ll \lambda)$
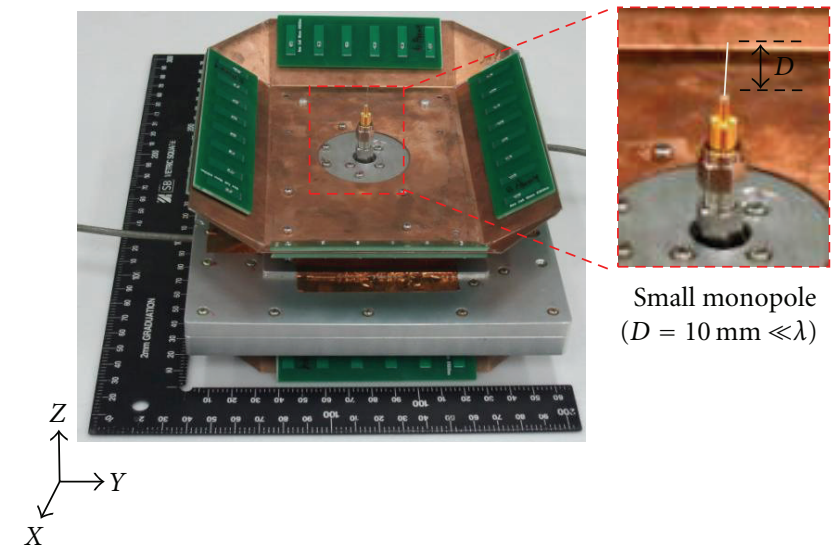

(b)

FIGURE 4: Small monopole antenna mounted on a conventional indoor repeater to measure the surface waves: (a) without FSS and (b) with FSS.

Since the proposed FSS has the periodic structure, the surface impedance can be calculated by using the Floquet modal analysis in incident wave mode directions $\left(\mathrm{E}_{\theta}, \mathrm{E}_{\Phi}\right)$ [13]. Figure 2 shows the geometry of a unit cell of the proposed FSS embedded into the FR4 substrate. It consists of the ground plane, a chip resistor $(620 \Omega)$, and a quarterwavelength resonator excited by the $\mathrm{E}_{\theta}$ mode. The height of a unit cell is $2 \mathrm{~mm}$. The size of ground plane is $30 \times 15 \mathrm{~mm}^{2}$.

Figure 3 shows the variation of the simulated surface impedance in the incident wave mode directions $\left(\mathrm{E}_{\theta}, \mathrm{E}_{\Phi}\right)$ with varying the width ( $W$, which is also the same as the distance between the unit cells in the FSS) of the unit cell ground plane. It is noticed that as the width $(W)$ decreases, the proposed unit cell of the FSS in the $\mathrm{E}_{\theta}$ mode direction is characterized by the HIS. However, varying the width
( $W=11,15,19 \mathrm{~mm}$ ), the surface impedance in the $\mathrm{E}_{\Phi}$ mode direction is barely changed and close to zero, so that the unit cell of the proposed FSS likely acts as a perfect conductor in the $\mathrm{E}_{\Phi}$ mode direction. Therefore, for an indoor repeater antenna, the surface impedance of the unit cell has to be matched with the free space impedance $\left(Z_{0}\right)$ in the TM mode $\left(\mathrm{E}_{\theta}\right.$ mode) excited by the obliquely incident wave of the angle $(\theta)$

$$
Z_{0}^{\mathrm{TM}}=\eta_{0} \cos \theta,
$$

where $\eta_{0}$ is the intrinsic wave impedance in free space. With the width $(W)$ of $15 \mathrm{~mm}$ and the inclined angle of $45^{\circ}$ at $\mathrm{E}_{\theta}$ mode direction, the surface impedance of the proposed unit cell is approximately matched with the wave impedance (266 $+\mathrm{j} 0 \Omega$ ) in the free space. 


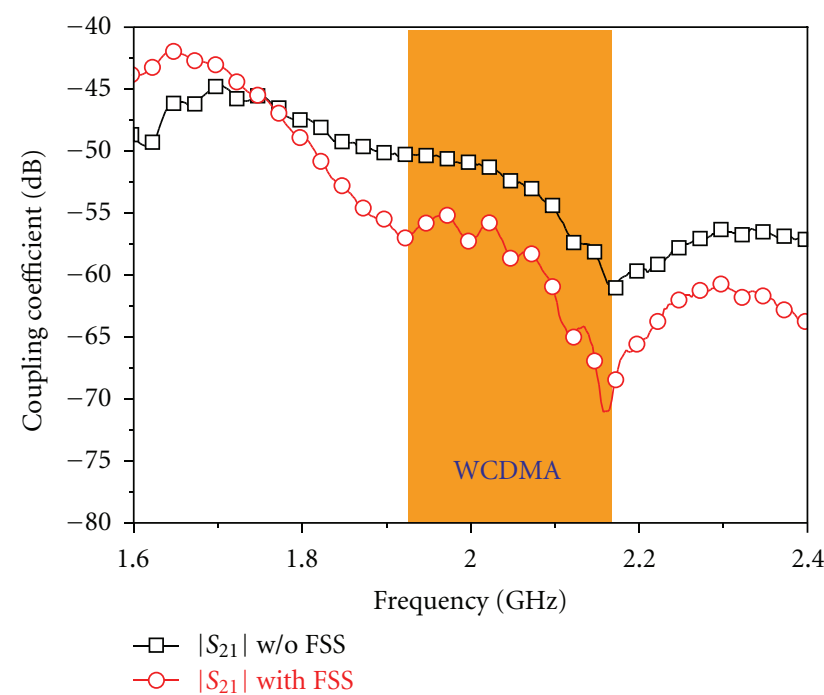

Figure 5: Measured dB magnitudes of $|\mathrm{S} 21|$.
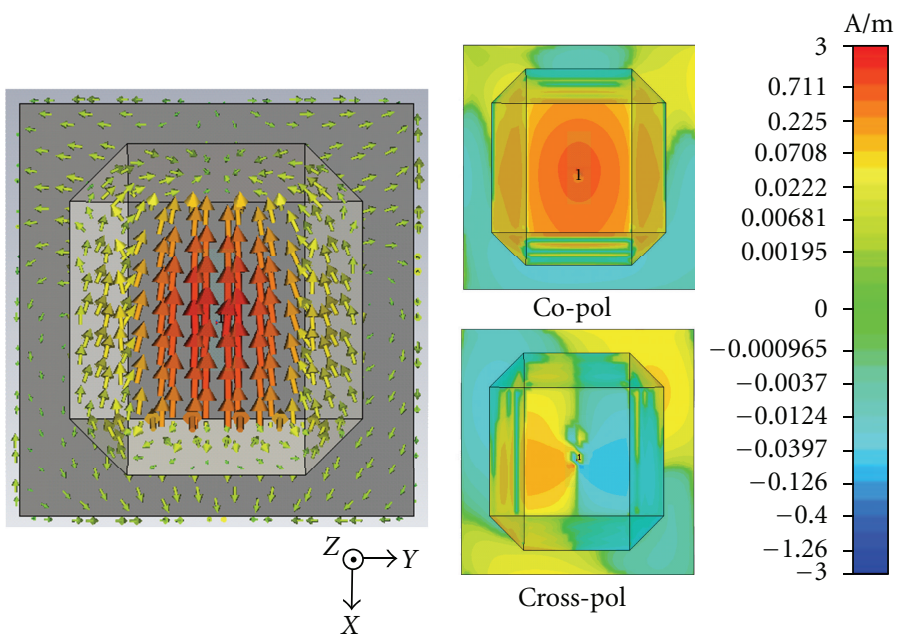

(a)
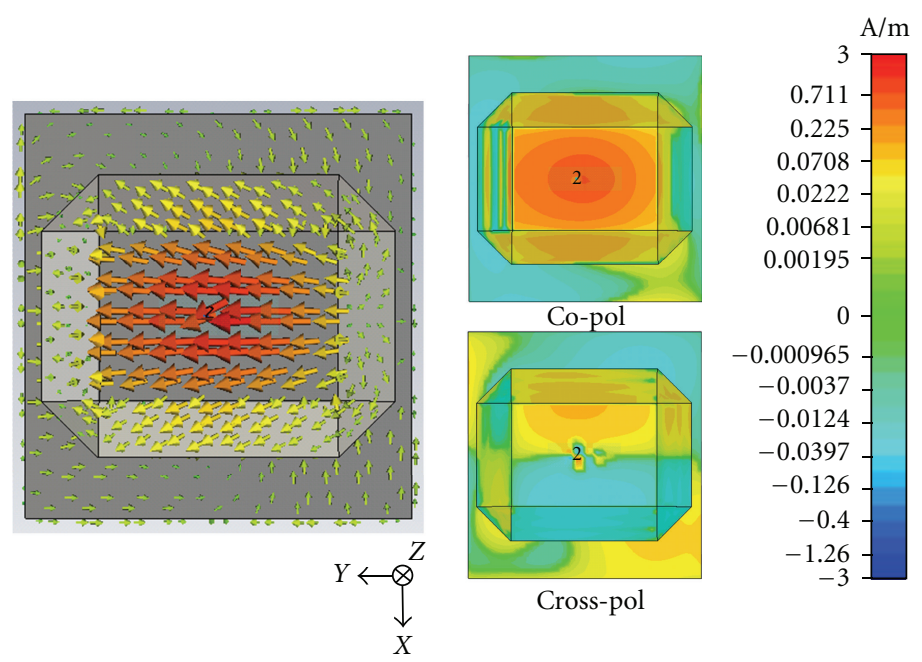

(b)

FIgURE 6: Surface current distributions on rectangle-bowl-type reflectors: (a) donor antenna and (b) service antenna. 


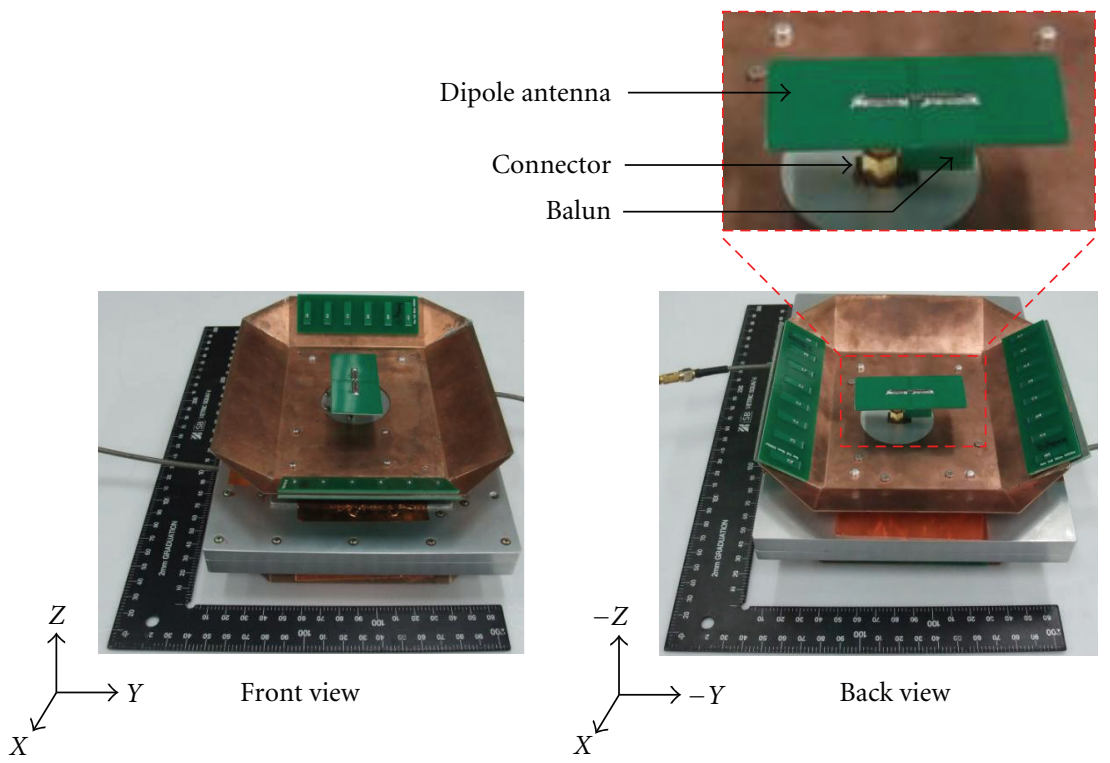

(a)
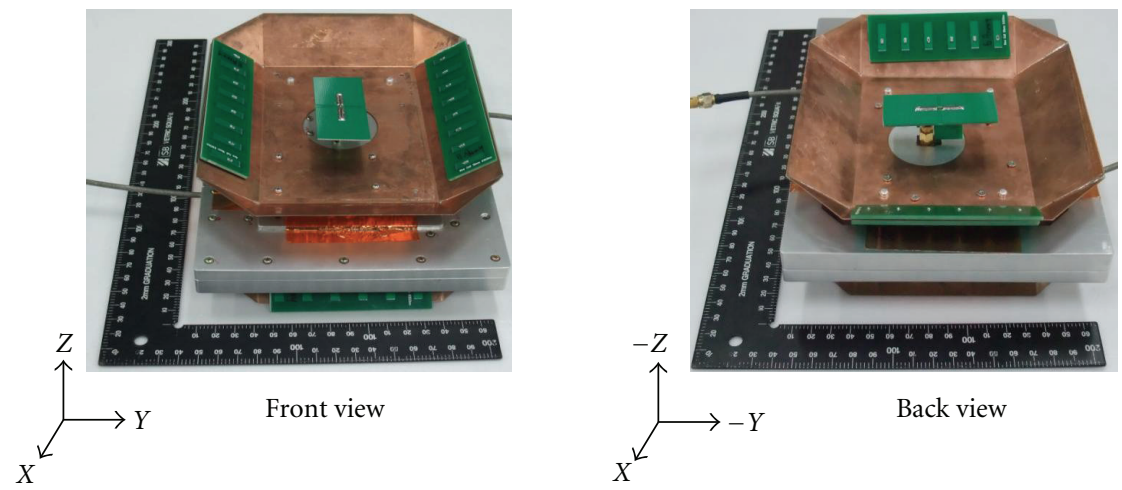

(b)

FIgURE 7: Configuration of proposed FSS in different locations: (a) Case I and (b) Case II.

\section{Isolation Measurement and Result}

To validate the performance of the proposed FSS, first, a small monopole for both the donor antenna and the service antenna mounted on a conventional indoor WCDMA repeater system as shown in Figure 4 is used to generate the surface waves on all side walls of the reflector, and then the isolation is measured. Figures 4(a) and 4(b) show a monopole antenna mounted on a conventional repeater system without and with the proposed FSS, respectively. Figure 5 shows the measured isolation between antennas as shown in Figure 4. It is noticed that the isolation can be considerably improved by the proposed FSS-embedded chip resistor (R) for the entire WCDMA band (1.92 $2.17 \mathrm{GHz}$ ). However, in general, to get the better isolation, a conventional repeater system uses two linearly polarized identical antennas (a donor antenna and a service antenna) orthogonally mounted on a reflector which stands back-toback. Even though two antennas are orthogonally mounted to get higher isolation between them, the surface currents that flow on the walls of the reflectors cause the crosspolarization as shown in Figure 6, so that it brings the unwanted mutual coupling between two antennas.

To further improve the isolation between them, the proposed FSS is embedded into the side walls of reflectors for a conventional indoor WCDMA repeater as shown in Figure 7. Even though, in Figure 4(b), the proposed FSS is embedded into all (four) side walls of each reflector, the effect of the isolation enhancement between orthogonally mounted antennas is conducted with varying the location of the FSS. Figure 7(a) (Case I) shows that the proposed FSS embedded into two side walls of each reflector is aligned along with the cross-polarization of each other antenna mounted on the other side of the reflector, and Figure 7(b) (Case II) shows that the proposed FSS embedded into two side walls of each reflector is aligned along with the cross-polarization of each antenna mounted on its own aperture reflector. Figure 8 shows the measured return loss and isolation for different locations of the FSS as shown in Figure 7. It is noticed that the location (Case II) of the 


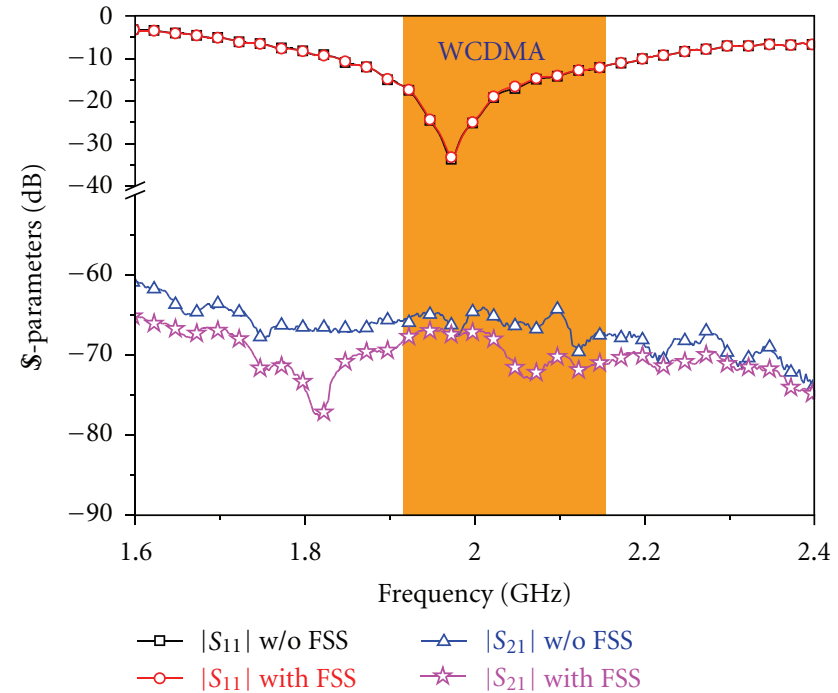

(a)

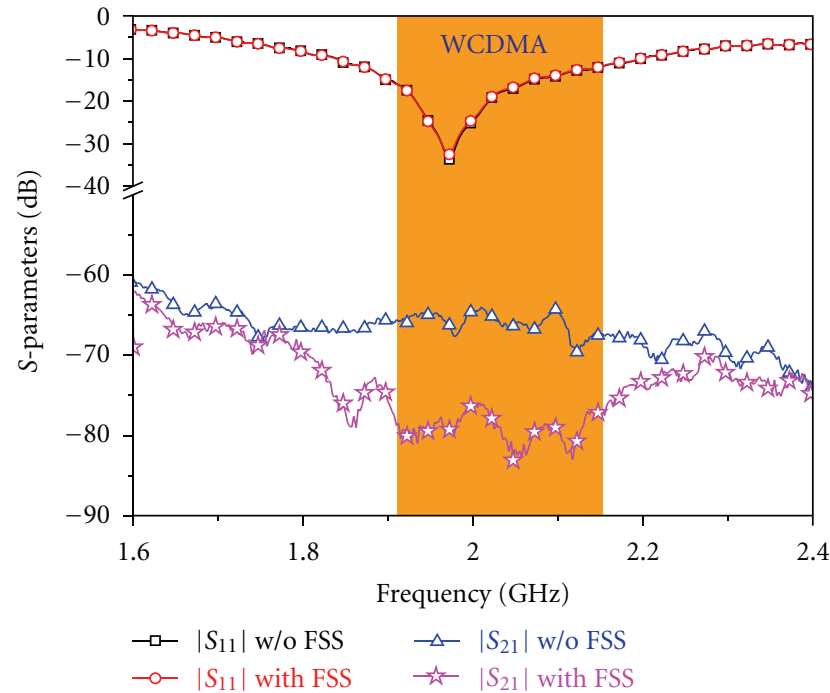

(b)

FIgURE 8: Measured dB magnitude of $|\mathrm{S} 11|$ and $|\mathrm{S} 21|$ : (a) Case I and (b) Case II shown in Figure 7.

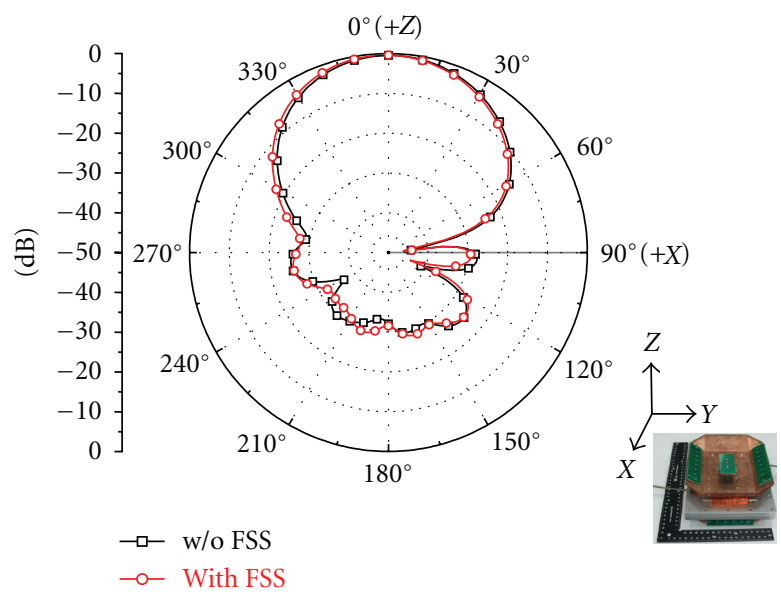

(a)

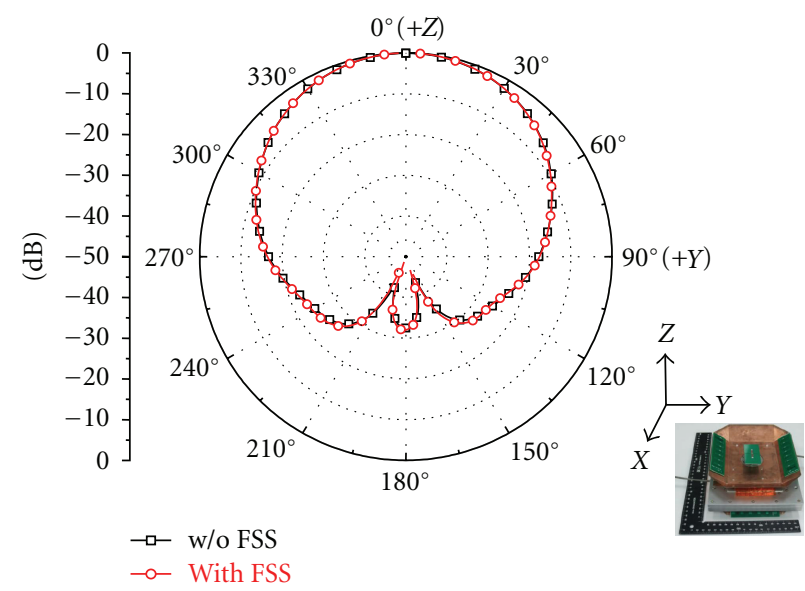

(b)

FIGURE 9: Measured radiation patterns of antenna (Case II in Figure 7) at $2.05 \mathrm{GHz}$ : (a) ZX-plane and (b) YZ-plane.

FSS shown in Figure 7(b) gives the better isolation by about $13 \mathrm{~dB}$ in the WCDMA band, and the resonant frequency of the antenna is barely changed by embedding the proposed FSS. Figure 9 shows the measured radiation patterns of the antenna (Case II) shown in Figure 7(b) at $2.05 \mathrm{GHz}$ with and without the proposed FSS. They are normalized by the peak gain of $9.5 \mathrm{dBi}$ (a typical value of the conventional indoor repeater antenna backed by the rectangle-bowltype reflectors), which is maintained without and with the proposed FSS.

\section{Conclusion}

The indoor repeater antenna with the proposed FSS has been implemented to obtain the higher isolation and maintain the peak gain. When the proposed FSS embedded into the side walls of the reflectors aligned along with the crosspolarization of each antenna, the isolation enhancement is about $13 \mathrm{~dB}$ in the WCDMA band without any sacrifice of the antenna radiation performance. The proposed FSS can practically be applied to a variety of wireless communication applications such as base-stations antenna and radars.

\section{Acknowledgment}

The present paper has been conducted by the Research Grant of Kwangwoon University in 2011.

\section{References}

[1] A. S. Mohd Marzuki, A. R. Abd Rahim, B. Mohmd, K. Khalil, A. Naemat, and A. Tee, "Antenna isolation considerations 
in WCDMA repeater deployment," in Proceedings of the International RF and Microwave Conference (RFM '6), pp. 347350, Putrajaya, Malaysia, September 2006.

[2] D. Sievenpiper, L. Zhang, R. F. Jimenez Broas, N. G. Alexöpolous, and E. Yablonovitch, "High-impedance electromagnetic surfaces with a forbidden frequency band," IEEE Transactions on Microwave Theory and Techniques, vol. 47, no. 11, pp. 2059-2074, 1999.

[3] O. Luukkonen, M. G. Silveirinha, A. B. Yakovlev, C. R. Simovski, I. S. Nefedov, and S. A. Tretyakov, "Effects of spatial dispersion on reflection from mushroom-type artificial impedance surfaces," IEEE Transactions on Microwave Theory and Techniques, vol. 57, no. 11, Article ID 5282500, pp. 26922699, 2009.

[4] S. Simms and V. Fusco, "Thin radar absorber using artificial magnetic ground plane," Electronics Letters, vol. 41, no. 24, pp. 1311-1313, 2005.

[5] Y.-Q. Li, Y.-Q. Fu, and N.-C. Yuan, "Characteristics estimation for high-impedance surfaces based ultrathin radar absorber," Microwave and Optical Technology Letters, vol. 51, no. 7, pp. 1775-1778, 2009.

[6] Q. Gao, Y. Yin, D. B. Yan, and N. C. Yuan, "Application of metamaterials to ultra-thin radar-absorbing material design," Electronics Letters, vol. 41, no. 17, pp. 936-937, 2005.

[7] C. Y. Chiu, C. H. Cheng, R. D. Murch, and C. R. Rowell, "Reduction of mutual coupling between closely-packed antenna elements," IEEE Transactions on Antennas and Propagation, vol. 55, no. 6, pp. 1732-1738, 2007.

[8] D. Ahn, J. S. Park, C. S. Kim, J. Kim, Y. Qian, and T. Itoh, "A design of the low-pass filter using the novel microstrip defected ground structure," IEEE Transactions on Microwave Theory and Techniques, vol. 49, no. 1, pp. 86-93, 2001.

[9] F. G. Zhu, J. D. Xu, and Q. Xu, "Reduction of mutual coupling between closely-packed antenna elements using defected ground structure," Electronics Letters, vol. 45, no. 12, pp. 601-602, 2009.

[10] S.-J. Kim, F. J. Harackiewicz, M.-J. Park et al., "Isolation enhancement between two closely mounted antennas for indoor repeater systems," Microwave and Optical Technology Letters, vol. 53, no. 3, pp. 697-700, 2011.

[11] O. Luukkonen, C. Simovski, G. Granet et al., "Simple and accurate analytical model of planar grids and high-impedance surfaces comprising metal strips or patches," IEEE Transactions on Antennas and Propagation, vol. 56, no. 6, pp. 1624-1632, 2008.

[12] S. A. Tretyakov and S. I. Maslovski, "Thin absorbing structure for all incidence angles based on the use of a high-impedance surface," Microwave and Optical Technology Letters, vol. 38, no. 3, pp. 175-178, 2003.

[13] I. Bardi, R. Remski, D. Perry, and Z. Cendes, "Plane wave scattering from frequency-selective surfaces by the finiteelement method," IEEE Transactions on Magnetics, vol. 38, no. 2 I, pp. 641-644, 2002. 

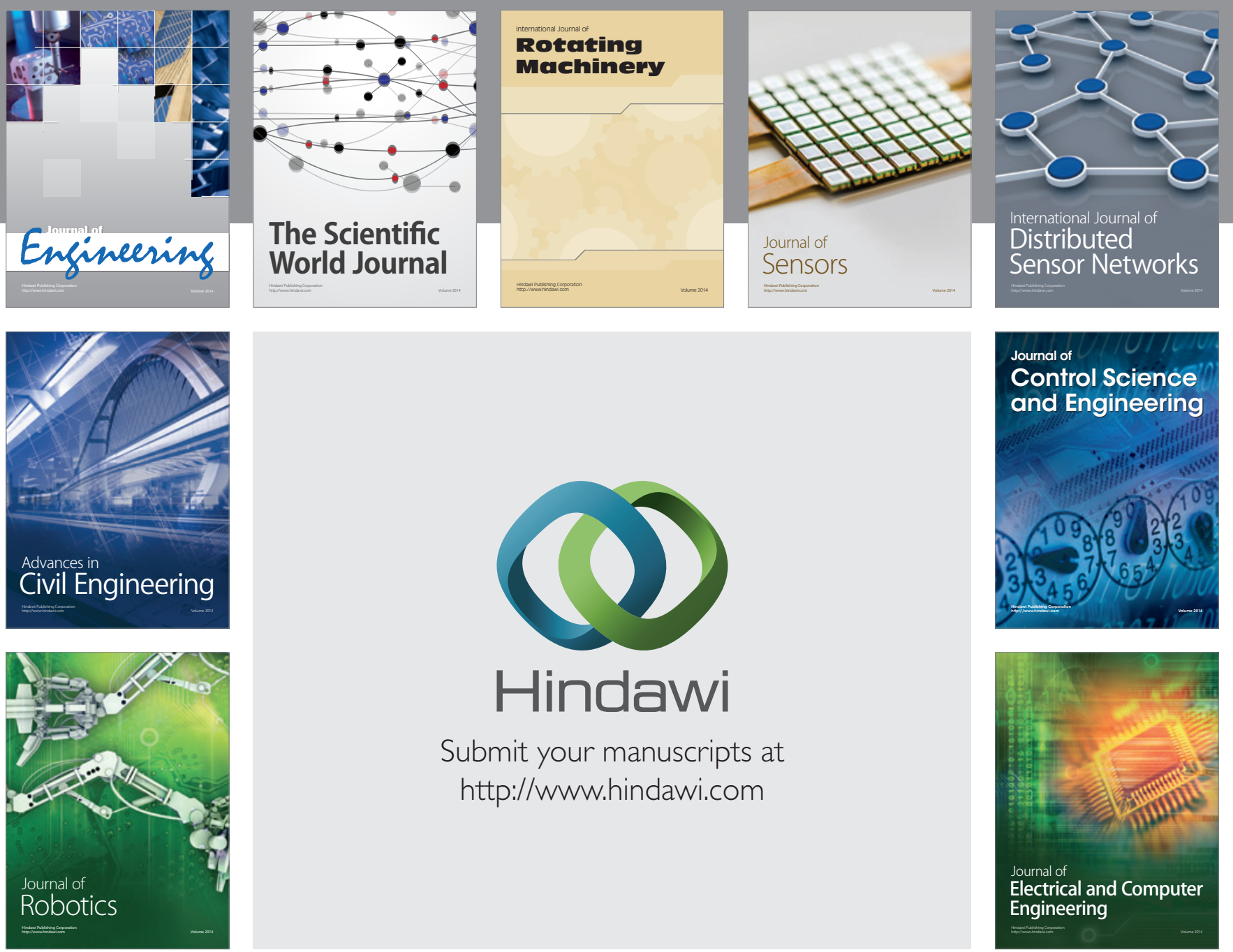

Submit your manuscripts at

http://www.hindawi.com
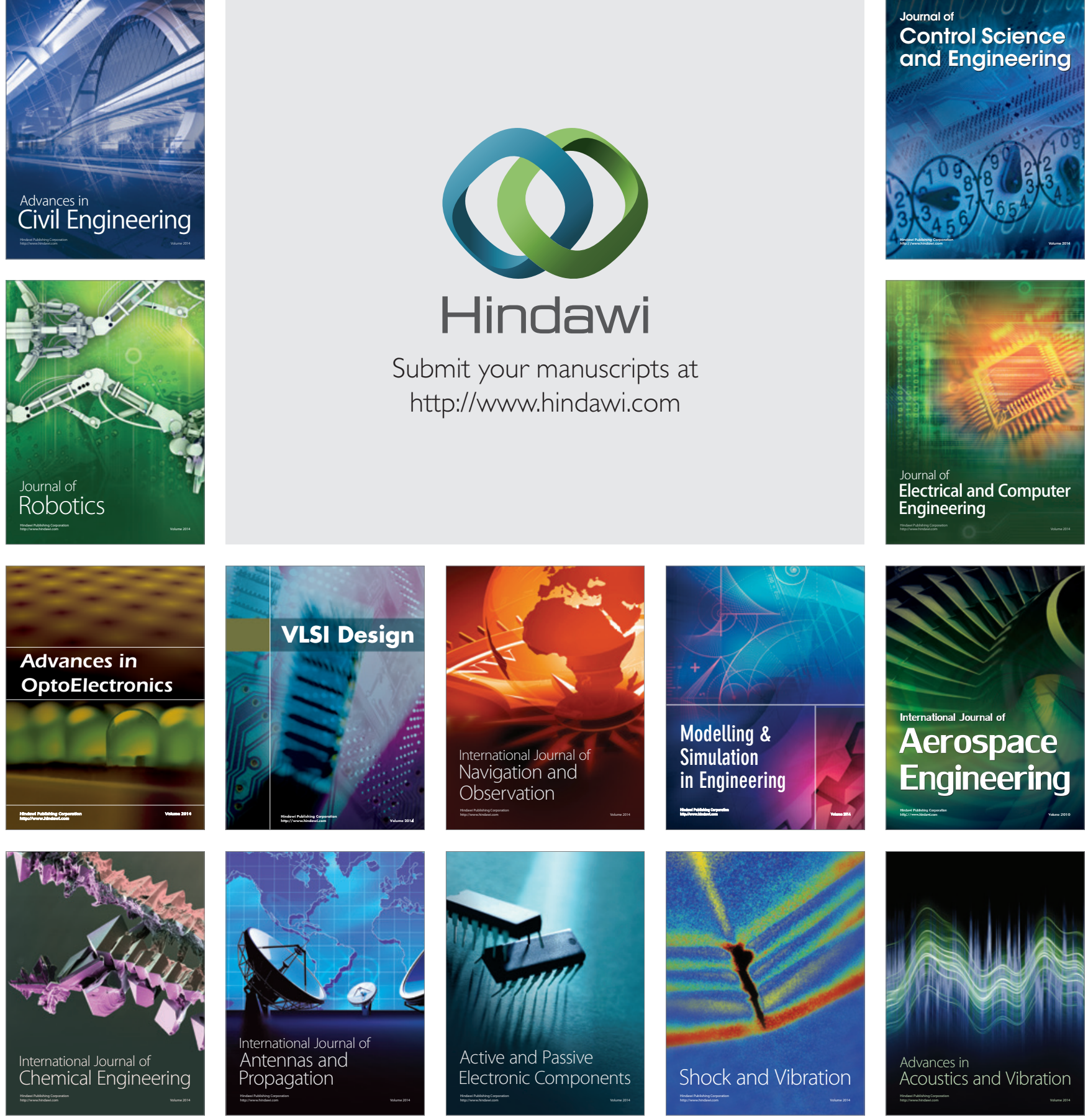